\title{
Insulin resistance and cardiovascular disease
}

\author{
Henry N. Ginsberg \\ Department of Medicine, Columbia University College of Physicians and Surgeons, 630 West 168th Street, \\ New York, New York 10032, USA. Phone: (212) 305-9562 ext. 69562; Fax: (212) 305-3213; E-mail: hng1@columbia.edu.
}

Insulin resistance means different things to different people. As other Perspectives in this series make clear, insulin resistance can be seen as a molecular and genetic mystery involving defective insulin signaling and glucose transport into cells. To me, on the other hand, insulin resistance represents a major underlying abnormality driving cardiovascular disease, the major cause of morbidity and mortality in much of the world. Because most of the work on insulin resistance has focused on its role in the pathophysiology of type 2 diabetes mellitus, a brief review of the history of the link between cardiovascular disease and insulin resistance is in order.

Margaret Albrink was probably the first investigator to identify a cluster of factors, including obesity and hypertriglyceridemia, that was associated with increased risk for coronary artery disease (CAD) (1). The groundbreaking development of the insulin radioimmunoassay by Berson and Yalow, and the subsequent observation that many diabetics were actually hyperinsulinemic, enabled Albrink and others, including Reaven and Farquhar and their colleagues (2), to begin to define the insulin resistance syndrome and its links to both hypertriglyceridemia and CAD. The next decades brought several prospective cohort studies in which hyperinsulinemia was often associated with CAD, at first in univariate and more recently in multivariate analyses. These efforts culminated recently in the demonstration by investigators in the Insulin Resistance Atherosclerosis Study (IRAS) of a link between a direct measure of insulin resistance itself and atherosclerosis (3). In addition, the 1970s brought a new understanding of protective roles of HDL (4). Together with the characterization of small dense LDLs in the 1980s, this advance led to the identification of a typical dyslipidemic pattern that is a central component of the insulin resistance syndrome. Another important addition to the complex was the observation by Welborn and colleagues in the mid-1960s that hypertension was commonly associated with hyperinsulinemia (5). Finally, the realization that individuals with insulin resistance both were hypercoagulable and had impaired fibrinolysis (6) added a pathologic basis for an increase in acute CAD events to the well accepted association of the insulin resistance syndrome with risk factors for atherosclerosis. As the components of the syndrome have increased, scientific interest and excitement, as well as the opportunities to investigate the links between insulin resistance and cardiovascular disease, have multiplied. In this Perspective I will attempt to provide an overview of what we do and do not know about the contribution of insulin resistance to the various components of the insulin resistance syndrome and to cardiovascular disease.

\section{Insulin resistance and dyslipidemia}

The scheme shown in Figure 1 offers a simple (even simplistic) view of lipid and lipoprotein metabolism that may be useful for the purposes of this Perspective. Insulin resistance at the level of the fat cell is shown as the initiating insult, leading to increased intracellular hydrolysis of triglycerides (TGs) and release of fatty acids into the circulation. It seems likely that insulin resistance in adipose tissue results from the interaction of multiple defective or less than optimally functioning genes with an environment that leads to increased pressure on the fat cell to store energy (see Kahn and Flier, this Perspective series, ref. 7). This interaction results in a fat cell that cannot meet the demands placed upon it. Whatever the molecular or environmental basis for insulin resistance in adipose tissue, the result is that FFA uptake by fat cells is decreased and/or FFA release from fat cells is increased, confronting the liver with increased availability of energy. Indeed, the hypertriglyceridemia of insulin resistance can be viewed as a Ping-Pong match between the hepatocytes and fat cells, where VLDLs and FFAs are the Ping-Pong balls carrying energy back and forth between the liver and the adipose tissue. The human disorders of lipodystrophy, characterized by marked insulin resistance and hypertriglyceridemia due to overproduction of VLDL, can be viewed as the ultimate loss of the sites for energy storage. Indeed, the hypertriglyceridemia in a lipodystrophic mouse model that has a genetic deficiency of adipose tissue was partially reversed by surgical implantation of normal adipose tissue (8). These findings suggest that the relationship between insulin resistance/hypertriglyceridemia and obesity is a parabolic one: Both the absence of fat and the abundance of fat are associated with increased fatty acid flux to the liver and subsequent increased secretion of VLDL.

Candidate genes that could underlie the increased flow of fatty acids include regulators of fatty acid 
uptake and storage by adipocytes, such as those for hormone-sensitive lipase (HSL) (9), lipoprotein lipase (LPL) (10), and complement component C3a (whose proteolytic product is the acylation-stimulating protein [ASP] [ref. 11]), as well as for various fatty acid transporters and binding proteins. Although some investigators have suggested links between HSL, LPL, and ASP, and either the insulin resistance/obesity syndrome or combined hyperlipidemia (which some believe is also the result of insulin resistance), no firm evidence yet exists linking these important proteins to the insulin resistance syndrome. Indeed, separate studies of a mouse in which the gene for ASP was deleted have resulted in contradictory results regarding its role in lipid metabolism $(11,12)$. On the other hand, recent data from mice carrying null mutations in the gene for the fatty acid transporter CD36 are quite revealing as to the consequences of the inability of fat cells to store fatty acids as TG. These animals had increased levels of plasma FFAs and hypertriglyceridemia, indicative of the response of the liver to an increased flux of energy from the periphery (13). Of interest, CD36 knock-out mice, which lack the fatty acid transporter in both adipose and muscle tissue, are not insulin-resistant. Indeed, they seem to be more insulin-sensitive, possibly as a result of diminished uptake of fatty acids by muscle. The dissociation of insulin resistance and TG metabolism in this model suggests a direct and critical role of increased plasma fatty acid flux to the liver in the genesis of hypertriglyceridemia. This conclusion is supported by work with transgenic mice overexpressing CD36 in muscle (14): these animals have low plasma levels of both fatty acids and TG but are insulin-resistant. These findings in gene-modified mouse models were paralleled by the observation that a strain of the spontaneously hypertensive rat (SHR) had mutations in $C D 36$ that appeared to be associated with insulin resistance $(15,16)$. However, a very recent report indicated that the original SHR line, which is insulin-resistant, has no defects in its CD36 gene (17). Further developments in this area of investigation are eagerly awaited.

In summary, the inability of insulin-resistant fat cells to store TG is very likely the initial step in the development of the dyslipidemia characteristic of insulin resistance. Importantly, this link between the fat cell and hepatic production of VLDL does not seem to be necessarily linked, in mouse models, to insulin resistance at the level of muscle. No candidate genes have been linked unequivocally to either the dyslipidemia or the insulin resistance in humans. Indeed, although the $C D 36$ gene appears to be an important candidate, the data from the $C D 36$ knock-out mice indicate to me that any CD36 defect would have to be specific to adipose tissue to cause both hypertriglyceridemia and insulin resistance.

Fatty acid flux and plasma VLDL TG levels. A large body of evidence has accumulated linking FFA availability to the assembly and secretion of VLDL by the liver (18). ApoB synthesis is constitutive under most physiologic conditions, with the assembly and secretion of apoBs regulated at the posttranslational stage by the availability of adequate core lipids (TG and cholesteryl esters) and the presence of microsomal triglyceride transfer protein (MTP) activity. In the absence of core lipids and/or MTP activity, newly syn-

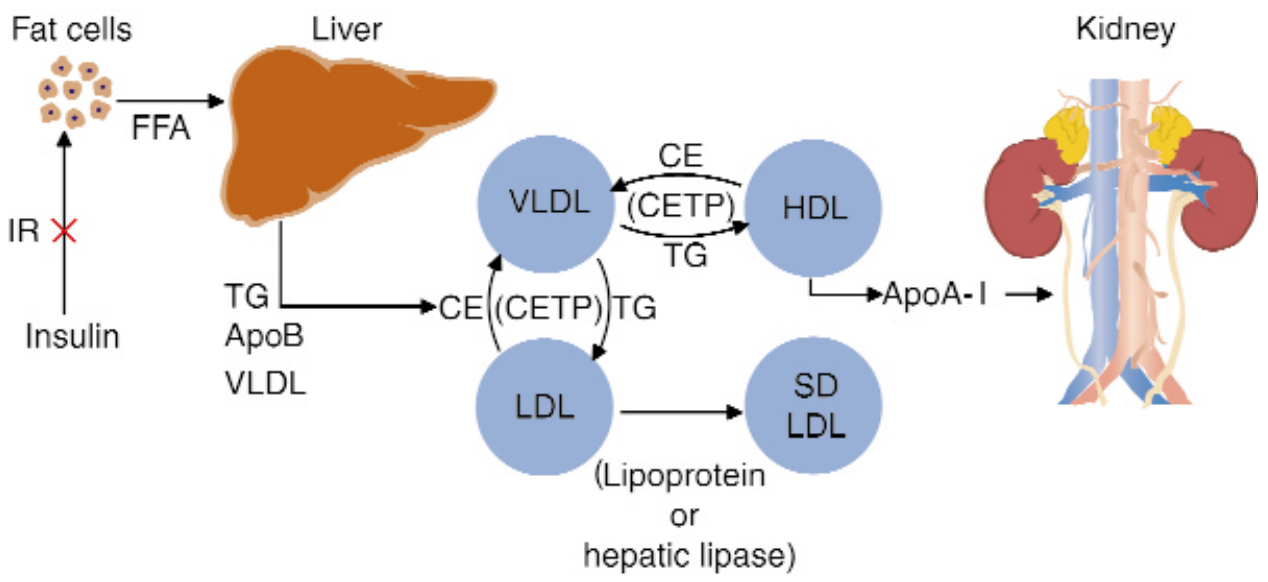

Figure 1

A simplified model relating insulin resistance to dyslipidemia and cardiovascular disease. Insulin resistance at the adipocyte results in increased release of fatty acids into the circulation. A similar accumulation of fatty acids could arise from defects in fatty acid transporters or intracellular binding proteins. Increased FFA flux to the liver stimulates the assembly and secretion of VLDL resulting in hypertriglyceridemia. In addition, VLDL stimulates the exchange of cholesteryl esters from both HDL and LDL for VLDL TG. ApoA-I can dissociate from TG-enriched HDL. This free apoA-I is cleared rapidly from plasma, in part by excretion through the kidney, thus reducing the availability of HDL for reverse cholesterol transport. TG-enriched LDL can undergo lipolysis and become smaller and more dense. Low levels of HDL and the presence of small dense LDL are each independent risk factors for cardiovascular disease. IR, insulin resistance; CE, cholesteryl ester; SD, small dense. 
thesized apoB is inefficiently translocated across the endoplasmic reticulum membrane and undergoes cotranslational and early posttranslational ubiquitination and degradation by the proteasome (19). This regulation, which appears to derive from the complex physical structure of apoB, particularly its long, highly hydrophobic $\beta$-sheet domains, provides a means whereby the liver can respond to the stress of increased lipid synthesis by immediately incorporating that lipid into VLDL and secreting it. In the absence of such need, the very large carrier protein, apoB, can be degraded as it is synthesized. Consistent with this model, increasing FFA flux has been shown to stimulate secretion of VLDL in humans (20).

The role of hyperinsulinemia in the assembly and secretion of VLDL, independent of its function as a marker of insulin resistance, has been controversial. At the cellular level, based on in vitro studies, short-term increases in insulin levels are associated with both increased TG synthesis and inhibition of apoB secretion. The inhibition of apoB secretion apparently occurs by a posttranslational degradative pathway; the site of this degradation remains to be determined. Longer incubations of cells with high concentrations of insulin, on the other hand, may increase VLDL secretion, perhaps because an insulin-resistant state develops in the cells after chronic treatment. In vivo studies in normal humans, using short-term hyperinsulinemic clamps, have supported an acute inhibitory function for hyperinsulinemia $(21,22)$. Importantly, obese subjects and subjects with type 2 diabetes appear to be resistant to these effects of insulin (23).

An interesting twist on the role of insulin in regulating plasma TG levels arose from studies of the apoCIII gene. ApoCIII plays an important role in regulating lipolysis of VLDL by LPL, and apoCIII-overexpressing transgenic mice have severe hypertriglyceridemia. Studies demonstrating the presence of an insulin response element (IRE) in the apoCIII promoter, and of suppressed gene expression by insulin, suggested that a specific type of insulin resistance might exist in hypertriglyceridemic individuals found to have sequence polymorphisms in this IRE (24). However, that association has not been found to be independent of the association of the SST polymorphism in the 3'untranslated region of apoCIII, and individuals with the SST polymorphism have higher TG levels irrespective of the status of their apoCIII IREs.

Cardiovascular disease and the dyslipidemia of insulin resistance. The available in vitro and in vivo data suggest that, as long as adequate insulin is available to prevent excessive lipid oxidation, FFA-driven lipid synthesis is the major determinant of VLDL TG secretion. Hyperinsulinemia probably is a marker of insulin resistance, rather than a major, direct contributor to the process. The basis for the rise in FFA flux in insulin resistance is probably oligogenic in nature, but whatever its ultimate cause, the rest of the dyslipidemic phenotype associated with insulin resistance follows once VLDL secretion increases (Figure 1). Hypertriglyceridemia, leading to low HDL cholesterol and increased small dense LDL particles, is due mainly to the actions of cholesteryl ester transfer protein (CETP) (25). In plasma, collisions between VLDL and HDL, in the presence of CETP, stimulate the transfer of VLDL TG to HDL in exchange for HDL cholesteryl esters. The resulting TG-enriched HDL becomes a good substrate for hepatic lipase (and possibly LPL), and the TG is hydrolyzed. This, in turn, generates a smaller HDL that must shed some of its surface, including apoA-I. ApoA-I is a small protein that can be filtered by the kidney and then degraded by renal tubular cells (26). In a similar fashion, intravascular collisions between VLDL and LDL allow for CETP-mediated exchange of VLDL TG for LDL cholesteryl esters. The succeeding hydrolysis of LDL TG generates small dense LDL particles.

Since both normoglycemic insulin-resistant individuals and patients with type 2 diabetes mellitus do not have higher LDL cholesterol levels than the general population, how does the resulting dyslipidemia increase the risk of insulin-resistant individuals to cardiovascular disease? I suggest that multiple aspects of their lipid profiles are atherogenic. First, not only are there increased levels of VLDL particles, which can enter the vessel wall and accumulate in atherosclerotic plaques $(27,28)$, but these VLDL are, by virtue of receiving CETP-transferred cholesteryl esters, able to deliver more cholesterol per particle to the vessel wall. Additionally, increased VLDL secretion can contribute to postprandial hyperlipidemia by providing competition for chylomicron clearance pathways; postprandial hyperlipidemia is independently associated with CAD (29). Second, reduced HDL cholesterol and apoA-I levels mean that there are fewer HDL particles engaged in cholesterol efflux from peripheral tissues, which is the first step in reverse cholesterol transport. Fewer HDL particles also mean that HDL cannot fulfill several proposed direct antiatherogenic actions at the vessel wall, including the role of HDL as an antioxidant. Krieger and colleagues recently identified scavenger receptor B1 (SRB1) (30), which appears to mediate the selective delivery of HDL cholesteryl esters to the liver (delivery of core lipid by the HDL particle without endocytosis and degradation of the whole particle), targeting that cholesterol for excretion via the biliary pathway. CETP-mediated transfer of HDL cholesteryl esters to VLDL not only may enrich an atherogenic lipoprotein with cholesterol but also can divert that cholesterol from the specific reverse cholesterol transport pathway. Theoretically, the reduced HDL cholesterol characteristic of insulin resistance could be the result of increased SRB1 expression; this is unlikely, however, because such an increase would 
likely reduce the risk of atherosclerosis (31). Finally, small dense LDL, first identified by Sniderman and colleagues (32) and then studied in depth by Krauss and Austin and their colleagues (33), may be more atherogenic than an equal number of larger more cholesteryl ester-rich LDL, because small dense LDL may be more liable to oxidation or may more readily penetrate and stick to the ECM of the artery wall.

\section{Insulin resistance and hypertension}

Although not as commonly associated with insulin resistance as dyslipidemia (34), hypertension most certainly adds significantly to the overall risk for cardiovascular disease if present. Unfortunately, the type of detailed mechanistic information describing the link between insulin resistance and dyslipidemia is not available for the link between hypertension and insulin resistance. Although high blood pressure and high insulin levels have been shown to be associated, independently of weight or body mass index, the link between obesity and both insulin resistance and hypertension further complicates the analysis of risks. It is important to reiterate, however, that the association between insulin resistance and hypertension is not as strong as between insulin resistance and dyslipidemia; only about $50 \%$ of hypertensive subjects are insulin-resistant.

Abnormalities in vasodilation and blood flow have been suggested to provide a link between hypertension and insulin resistance. Insulin given intravenously causes vasodilation in normal subjects, and this response is deficient in obese, insulin-resistant subjects and in patients with type 2 diabetes mellitus (35). Baron and his colleagues have suggested that this defect appears to reside in a failure of insulin to stimulate the secretion of nitric oxide (NO) by endothelial cells. Of interest, increased plasma FFAs can inhibit vasodilation in response to methylcholine, which acts via $\mathrm{NO}(36)$. The link between endothelial dysfunction and the hypertension of the insulin resistance syndrome is enticing because of the possibility that defective vasodilation actually produces insulin resistance. Blood flow is a key variable in the regulation of glucose uptake by tissue beds; if defective small arteriolar vasodilation is a characteristic of insulin-resistant individuals, could this be associated with decreased insulin-mediated delivery of glucose to muscle? The Heart Outcomes Prevention Evaluation (HOPE) Study investigators recently made the intriguing observation that treatment with an angiotensin-converting enzyme inhibitor was associated with reduced incidence of new-onset diabetes (37), perhaps indicating that inhibiting vasoconstriction by inhibiting angiotensin formation improves insulin sensitivity and thereby delays the onset of type 2 diabetes. However, the ability of physiologically relevant insulin levels to cause vasodilation is contro- versial; in some studies, low levels of insulin that did not increase forearm blood flow did cause increased glucose uptake.

It is well known that hyperinsulinemia can result in increased reabsorption of sodium and water by kidney tubular cells (38), and this can be associated with a volume-dependent hypertension. However, it is not clear how often volume-dependent hypertension is present in insulin-resistant individuals and patients with type 2 diabetes. Another candidate as causative for hypertension in the insulin-resistant patient is overactivity of the sympathetic nervous system (39). Much evidence suggests that the sympathetic system is overactive in obese and insulin-resistant individuals, but it has not been shown that this is a primary defect in these individuals.

\section{Insulin resistance and clotting}

Numerous epidemiologic and clinical studies have provided evidence of the importance of several factors integral to clotting and fibrinolysis, including fibrinogen, factor VII, and plasminogen activator inhibitor 1 (PAI-1), for the risk of developing cardiovascular disease. It has also been demonstrated that these factors are increased in individuals with insulin resistance. The basis for increased levels of fibrinogen in the insulin resistance syndrome is not clear, but it may be related to obesity. Factor VII activity has been shown to increase during postprandial hyperlipidemia (40), suggesting a risk for acute CAD events after consumption of a high-fat meal. PAI-1 was shown by Hamsten et al. (41) to be a marker for risk of premature $\mathrm{CAD}$, and the link between elevated PAI1 and insulin resistance has been studied extensively in tissue culture models. Both hepatic cells and endothelial cells respond to increased levels of insulin by synthesizing and secreting more PAI-1. Incubation of endothelial cells with VLDL also increases PAI-1 synthesis and secretion; a putative VLDL response element, recently identified in the gene for PAI-1, may be responsible for this induction (42).

\section{Insulin resistance, hyperinsulinemia and atherogenesis}

Does hyperinsulinemia per se contribute to the insulin resistance syndrome? Is it just the physiologic consequence of diminished insulin-mediated glucose uptake in muscle and therefore only a marker of insulin resistance, or does it somehow promote the insulin resistance syndrome? The answer may differ for the various aspects of this syndrome. As noted above, insulin may be permissive with respect to VLDL assembly and secretion and the consequent dyslipidemia, in that adequate insulin is needed to suppress fatty acid oxidation, but it probably does not play a direct pathophysiologic role. Insulin's role in hypertension may be somewhat more direct, with hyperinsulinemia potentially leading to salt and water reten- 
tion. Hyperinsulinemia may also directly inhibit fibrinolysis in individuals with insulin resistance; in addition to the tissue culture studies noted above, PAI-1 secretion is stimulated by the intravenous infusion of insulin in humans (43). On the other hand, high levels of VLDL also stimulate increased PAI-1 synthesis and secretion by cultured cells, a process that appears to be sensitive to a specific PAI-1 genotype (44).

The findings by several groups that insulin resistance seems to spare the MAP kinase component of the insulin signaling pathway also suggest that insulin could be atherogenic by stimulating division and migration of vascular smooth muscle cells. Insulin's effects on PAI-1 expression may be mediated by the MAP kinase pathway as well. Indeed, thiazolidinediones and other peroxisome proliferator-activated receptor $\gamma$ (PPAR $\gamma$ ) ligands inhibit insulin signaling downstream of MAP kinase, and these agents inhibit smooth muscle cell proliferation (45) and migration (46).

The controversy about the role of insulin itself as an atherogenic molecule is not simply an academic exercise, because it has clinical implications: if insulin is atherogenic, insulin therapy is a poor choice for patients with insulin resistance and either absolute or relative insulin hyperinsulinemia. However, the result of the United Kingdom Prospective Diabetes Study (UKPDS), in which insulin and sulfonylurea treatments were associated with a nearly statistically significant reduction of CAD events $(P=0.052)$, provides strong evidence against a direct atherogenic effect of insulin (47) and supports the use of insulin and insulin secretogogues as hypoglycemic agents. These clinical data are supported by a study in cholesterol-fed rabbits, in which administration of exogenous insulin for 24 weeks did not increase atherosclerosis (48).

\section{Insulin resistance and the artery wall}

There is increasing evidence that insulin resistance may be directly atherogenic. The results of IRAS suggest a direct relationship between insulin resistances, as measured by the frequently sampled intravenous glucose tolerance test and carotid artery intima/media thickness, even after adjusting for several associated risk factors (3). Although those results could simply indicate that there are other risk factors for atherosclerosis that were not measured in IRAS, they can also be taken to indicate direct effects of impaired insulin action at the level of endothelial or vascular smooth muscle cells. The efficacy of thiazolidinediones in ameliorating endothelial dysfunction in individuals with the insulin resistance syndrome is consistent with the latter possibility. Finally, as described above, insulin signaling pathways in smooth muscle cells may be differentially affected in the insulin resistance syndrome so that hyperinsulinemia, resulting from insulin resistance in muscle, fat, and liver, may stimulate proatherogenic pathways in vascu- lar smooth muscle cells. The potential role of PPAR $\gamma$ receptors in those cells offers new targets for affecting atherogenesis directly in insulin-resistant patients.

\section{Summary}

Clearly, insulin resistance is not simply a problem of deficient glucose uptake in response to insulin, but a multifaceted syndrome that increases significantly the risk for cardiovascular disease. The links between insulin resistance and the associated dyslipidemia, hypertension, hypercoagulability, and atherosclerosis are numerous and complex. This complexity derives both from the almost certain multiple causes of the insulin resistance syndrome and from the interaction of genes predisposing to insulin resistance with other genes that have their own, independent impact on lipid metabolism, blood pressure regulation, coagulation, and artery wall biology. Nonetheless, I suggest that dysregulation of fatty acid metabolism plays a central role in the development of this phenotype. Thus, the association between insulin resistance and dyslipidemia is clearly initiated by increased FFA release from, or defective uptake of FFAs into, adipocytes. Recent studies linking fatty acids to endothelial dysfunction, together with the clear role of VLDL in the stimulation of PAI1 , further support the view that dysregulation of fatty acid metabolism sits close to the center of the pathophysiology of the insulin resistance syndrome, at least as it relates to risk for cardiovascular disease. and Wood, P.D.S. 1980. Intercorrelations among high density lipoproteins, obesity, and triglycerides in a normal population. Lipids. 15:668-678.

2. Reaven, G.M., Lerner, R.L., Stern, M.P., and Farquhar, J.W. 1967. Role of insulin in endogenous hypertriglyceridemia. J. Clin. Invest. 46:1756-1767.

3. Howard, G., et al. 1996. Insulin sensitivity and atherosclerosis. Circulation. 93:1809-1817.

4. Miller, G.J., and Miller, N.E. 1975. Plasma high density lipoprotein concentration and development of ischaemic heart disease. Lancet. 1:16-19.

5. Welborn, T.A., Breckenridge, A., Rubinstein, A.H., Dollery, C.T., and Fraser, T.R. 1966. Serum-insulin in essential hypertension and in peripheral vascular disease. Lancet. 1:1336-1337.

6. Juhan-Vague, I., Alessi, M., and Vague, P. 1991. Increased plasma PAI1 levels: a possible link between insulin resistance and atherothrombosis. Diabetologia. 34:457-462.

7. Kahn, B.B., and Flier, J.S. 2000. Obesity and insulin resistance. J. Clin. Invest. 106:473-481.

8. Gavrilova, O., et al. 2000. Surgical implantation of adipose tissue reverses diabetes in lipoatrophic mice. J. Clin. Invest. 105:271-278.

9. Klannemark, M., Orho, M., Langin, D.L.H., Holm, C., and Reynisdottir, S. 1998. The putative role of the hormone-sensitive lipase gene in the pathogenesis of Type II diabetes mellitus and abdominal obesity. Diabetologia. 41:1516-1522.

10. Babirak, S.P., Brown, B.G., and Brunzell, J.D. 1992. Familial combined hyperlipidemia and abnormal lipoprotein lipase. Arterioscler. Thromb. 10:1176-1183.

11. Murray, I., Havel, P.J., Sniderman, A.D., and Cianflone, K. 2000. Reduced body weight, adipose tissue, and leptin levels despite increased energy intake in female mice lacking acylation-stimulating protein. Endocrinology. 141:1041-1049.

12. Wetsel, R.A., Kildsgaard, J., Zsigmond, E., Liao, W., and Chan, L. 1999. Genetic deficiency of acylation stimulating protein (ASP(C3ades-Arg)) does not cause hyperapobetalipoproteinemia in 
mice. J. Biol. Chem. 274:19429-19433.

13. Febbraio, M., et al. 1999. A null mutation in murine CD36 reveals an important role in fatty acid and lipoprotein metabolism. J. Biol. Chem. 274:19055-19062.

14. Ibrahimi, A., et al. 1999. Muscle-specific overexpression of FAT/CD36 enhances fatty acid oxidation by contracting muscle, reduces plasma triglycerides and fatty acids, and increases plasma glucose and insulin. J. Biol. Chem. 274:26761-26766.

15. Aitman, T.J., et al. 1999. Identification of Cd36 (Fat) as an insulinresistance gene causing defective fatty acid and glucose metabolism in hypertensive rats. Nat. Genet. 21:76-83.

16. Pravenec, M., et al. 1999. Genetics of Cd36 and the clustering of multiple cardiovascular risk factors in spontaneous hypertension. J. Clin. Invest. 103:1651-1657.

17. Gotoda, T., et al. 1999. Absence of Cd36 mutation in the original spontaneously hypertensive rats with insulin resistance. Nat. Genet. 22:226-228.

18. Dixon, J.L., and Ginsberg, H.N. 1993. Regulation of hepatic secretion of apolipoprotein B-containing lipoproteins: information obtained from cultured liver cells. J. Lipid Res. 34:167-179.

19. Fisher, E., et al. 1997. Apoprotein B100, an atypical secretory protein, can be degraded by a cytosolic pathway involving heat shock protein 70 and proteasomes. J. Biol. Chem. 272:20427-20434.

20. Lewis, G.F. 1997. Fatty acid regulation of very low density lipoprotein (VLDL) production. Curr. Opin. Lipidol. 8:146-153.

21. Lewis, G.F., Uffelman, K.D., Szeto, L.W., and Steiner, G. 1993. Effects of acute hyperinsulinemia on VLDL triglyceride and VLDL apoB production in normal weight and obese individuals. Diabetes. 42:833-842.

22. Malmstrom, R., et al. 1998. Effects of insulin and acipimox on VLDL1 and VLDL2 apolipoprotein B production in normal subjects. Diabetes. 47:779-787.

23. Malmstrom, R., et al. 1997. Defective regulation of triglyceride metabolism by insulin in the liver in NIDDM. Diabetologia. 40:454-462.

24. Dammerman, M., Sandkuijl, L.A., Halaas, J.L., Chung, W., and Breslow, J.L. 1993. An apolipoprotein CIII haplotype protective against hypertriglyceridemia is specified by promoter and $3^{\prime}$ untranslated region polymorphisms. Proc. Natl. Acad. Sci. USA. 90:4562-4566.

25. Bruce, C., Chouinard, R.A., Jr., and Tall, A.R. 1998. Plasma lipid transfer proteins, high-density lipoproteins, and reverse cholesterol transport. Annu. Rev. Nutr. 18:297-330.

26. Horowitz, B.S., et al. 1993. Increased plasma and renal clearance of an exchangeable pool of apolipoprotein A-I in subjects with low levels of high density lipoprotein cholesterol. J. Clin. Invest. 91:1743-1752.

27. Nordestgaard, B.G., Tybjaerg-Hansen, A., and Lewis, B. 1992. Influx in vivo of low density, intermediate density, and very low density lipoproteins into aortic intimas of genetically hyperlipidemic rabbits. Roles of plasma concentrations, extent of aortic lesion, and lipoprotein particle size as determinants. Arterioscler. Thromb. 12:6-18.

28. Rapp, J.H., et al. 1994. Triglyceride-rich lipoproteins isolated by selected-affinity anti-apolipoprotein B immunosorption from human atherosclerotic plaque. Arterioscler. Thromb. 14:1767-1774.

29. Ginsberg, H.N., et al. 1995. Association of postprandial triglyceride and retinyl palmitate responses with newly diagnosed exerciseinduced myocardial ischemia in middle-aged men and women. Arterioscler. Thromb. Vasc. Biol. 15:1829-1838.

30. Acton, S., et al. 1996. Identification of scavenger receptor SR-BI as a high density lipoprotein receptor. Science. 271:460-461.
31. Arai, T., Wang, N., Bezouevski, M., Welch, C., and Tall, A.R. 2000. Decreased atherosclerosis in heterozygous low density lipoprotein receptor-deficient mice expressing the scavenger receptor BI transgene. J. Biol. Chem. 274:2366-2371.

32. Sniderman, A.D., et al. 1982. Association of hyperapobetalipoproteinemia with endogenous hypertriglyceridemia and atherosclerosis. Ann. Intern. Med. 97:833-839.

33. Austin, M.A., King, M.D., Vranizan, K.M., and Krauss, R.M. 1990. Atherogenic lipoprotein phenotype. A proposed genetic marker for coronary heart disease risk. Circulation. 82:495-506.

34. Bonora, E., et al. 1998. Prevalence of insulin resistance in metabolic disorders: the Bruneck Study. Diabetes. 47:1643-1649.

35. Laakso, M., Edelman, S.V., Brechtel, G, and Baron, A.D. 1990. Decreased effect of insulin to stimulate skeletal muscle blood flow in obese man. A novel mechanism for insulin resistance. J. Clin. Invest. 85:1844-1852.

36. Steinberg, H.O., et al. 1997. Elevated circulating free fatty acid levels impair endothelium-dependent vasodilation. J. Clin. Invest. 100:1230-1239.

37. Yusuf, S., et al. 2000. Effects of an angiotensin-converting-enzyme inhibitor, ramipril, on cardiovascular events in high-risk patients. The Heart Outcomes Prevention Evaluation Study Investigators. $N$. Engl. J. Med. 342:145-153.

38. Defronzo, R.A., Cooke, C.R., Andres, R., Faloona, G.R., and Davis, P.J. 1975. The effect of insulin on renal handling of sodium, potassium, calcium, and phosphate in man. J. Clin. Invest. 55:845-855.

39. Landsberg, L. 1999. Insulin resistance and hypertension. Clin. Exp. Hypertens. 21:885-894.

40. Miller, G.J. 1995. Lipoproteins and thrombosis: effects of lipid lowering. Curr. Opin. Lipidol. 6:38-42.

41. Hamsten, A., Wiman, B., de Faire, U., and Blomback, M. 1985. Increased plasma levels of a rapid inhibitor of tissue plasminogen activator in young survivors of myocardial infarction. N. Engl. J. Med. 313:1557-1563.

42. Eriksson, P., Nilsson, L., Karpe, F., and Hamsten, A. 1998. Very-lowdensity lipoprotein response element in the promoter region of the human plasminogen activator inhibitor-1 gene implicated in the impaired fibrinolysis of hypertriglyceridemia. Arterioscler. Thromb. Vasc. Biol. 18:20-26.

43. Calles-Escandon, J., Mirza, S.A., Sobel, B.E., and Schneider, D.J. 1998. Induction of hyperinsulinemia combined with hyperglycemia and hypertriglyceridemia increases plasminogen activator inhibitor 1 in blood in normal human subjects. Diabetes. 47:290-293.

44. Li, X.N., et al. 1997. Genotype-specific transcriptional regulation of PAI-1 expression by hypertriglyceridemic VLDL and Lp(a) in cultured human endothelial cells. Arterioscler. Thromb. Vasc. Biol. 17:3215-3223.

45. Law, R.E., et al. 1996. Troglitazone inhibits vascular smooth muscle cell growth and intimal hyperplasia. J. Clin. Invest. 98:1897-1905.

46. Marx, N., Schonbeck, U., Lazar, M.A., Libby, P., and Plutzky, J. 1998. Peroxisome proliferator-activated receptor gamma activators inhibit gene expression and migration in human vascular smooth muscle cells. Circ. Res. 83:1097-1103.

47. 1998. Intensive blood-glucose control with sulphonylureas or insulin compared with conventional treatment and risk of complications in patients with type 2 diabetes (UKPDS 33). UK Prospective Diabetes Study (UKPDS) Group. Lancet. 352:837-853.

48. Nordestgaard, B.G., Agerholm-Larson, B., and Stender, S. 1997. Effect of exogenous hyperinsulinaemia on atherogenesis in cholesterol-fed rabbits. Diabetologia. 40:512-520. 